\title{
Green energy generation via optical laser pressure initiated nonthermal nuclear fusion
}

\author{
Heinrich Hora $\odot$, a,b Alex Fuerbach $\odot,{ }^{\text {c }}$ Francois Ladouceur $\odot,{ }^{\text {a }}$ and \\ Warren McKenzie ${ }^{a, b}, *$ \\ ${ }^{a}$ University of New South Wales, Sydney, Australia \\ ${ }^{b}$ HB11 Energy Holding Pty Ltd., Sydney, Australia \\ ${ }^{c}$ Macquarie University, Department of Physics and Astronomy, Sydney, Australia
}

\begin{abstract}
In a recent report, the National Academies of Science, Engineering, and Medicine (NASEM) recommends that "the United States should start a national program of accompanying research and technology leading to the construction of a compact pilot plant that produces electricity from fusion at the lowest possible capital cost." It is generally acknowledged that a decarbonization of the world's energy system is unavoidable to combat climate change. While an exothermic chemical reaction such as the combustion of fossil fuels produces an energy of $<1 \mathrm{eV}$ per molecule, a nuclear fusion reaction is an attractive alternative as it releases 10 million times more energy. To date, considerable effort has been devoted to research involving the fusion between the nuclei of the two heavy isotopes of hydrogen: deuterium (D) and tritium (T). However, the main roadblock for the adoption of this technology is the need to heat the fuel to temperatures in the order of 50 million Kelvin and to keep it stable under extreme pressure conditions. Recent results show that this difficulty can be overcome by utilizing the nonthermal radiation pressure that can be generated via chirped-pulse amplifier laser systems and can trigger the fusion of hydrogen and boron-11 nuclei, producing clean energy in the form of kinetic alpha particles, thus sidestepping nuclear radiation problems due to the aneutronic nature of the process. () The Authors. Published by SPIE under a Creative Commons Attribution 4.0 International License. Distribution or reproduction of this work in whole or in part requires full attribution of the original publication, including its DOI. [DOI: 10.1117/1.OE.61.2.021004]
\end{abstract}

Keywords: nuclear fusion; chirped-pulse lasers; radiation pressure; hydrogen-boron fusion.

Paper 20210760SS received Jul. 17, 2021; accepted for publication Oct. 12, 2021; published online Nov. 10, 2021.

\section{Controlled Thermonuclear Fusion for Electricity Generation}

Human progress always was and still is dependent on the availability of an abundant source of energy. The rapid rate of climate change and the resulting massive impacts on the environment that are caused by our reliance on fossil fuels means that the development of clean energy sources has become one of the most pressing issues of our times. This is acknowledged by the United States National Academy of Science, Engineering and Medicine (NASEM) who clearly recommended in a recent report to focus on nuclear fusion research, a technology that has enormous potential to play an essential role in the future. ${ }^{1}$ The urgency of this problem is also reflected in the medium- to long-term energy projections that the International Energy Agency regularly provides using the so-called World Energy Model. According to the World Energy Outlook 2020, global energy needs rise by about $19 \%$ to 2040 . Without anticipated decreases in energy intensity of GDP, global energy needs would almost double by 2040 while the effective generation capacity of fossil fuels should be rapidly decreasing. ${ }^{2}$ The fact that the growing gap between total energy production and total energy consumption has the potential to trigger a global energy crisis was predicted as far back as 2010 in the Total Energy Outlook of the US Energy Information Administration. ${ }^{3}$ A deeper analysis of the motivation of the climate regulations shows they are significantly motivated by the need to reduce energy consumption to provide more time for development of alternative technologies of energy generation, including novel technologies based on fusion.

*Address all correspondence to Warren McKenzie, Warren.McKenzie@hb11.energy 
Nuclear fusion is a topic that is too general and too complex for it to be discussed in detail in this review, which therefore aims at complementing the existing literature. In this article, we will focus on one specific aspect of fusion research, namely the question of the involved heat generation and what modern research into nonthermal optical pressures can offer as a possible and additional new direction. There is mounting evidence that the use of high-power chirped-pulse laser systems can accelerate hydrogen atoms to such high energies as to initiate efficient fusion reactions without the need of heating the fuel to extreme temperatures of tens of million degrees.

When Lord Rutherford discovered nuclear physics over 120 years ago, he realized that the exchanged energy per nuclear reaction is about 10 million times higher than the energy involved in chemical reactions. This is the reason why a piece of coal can be ignited by a match, but nuclear reactions can only be initiated via particle collisions with about 10 million times higher energies. For the reaction that powers the Sun, i.e., the fusion of two hydrogen nuclei into a single helium nucleus with an enormous net-positive energy gain of $17.6 \mathrm{MeV}$, the optimal reaction rates occur at temperatures of many dozens of million ${ }^{\circ} \mathrm{C}$. In contrast, the energy exchange for burning coal corresponds to thermal energies much less in the electron-volt $(\mathrm{eV})$ range.

In the 1940s and 1950s, the possibility of initiating explosive nuclear fission and fusion reactions was demonstrated, and it was then obvious to ask how these processes can be utilized in a controlled way in a reactor for energy generation, to eliminate the need for burning of fossil fuels. As discussed in the NASEM report, ${ }^{1}$ early experiments all concentrated on low-density fusion reactors at equilibrium where the fuel must not only be heated to extreme temperature but must also be kept stable under intense pressure, hence dense enough and confined for long enough to allow the nuclei to fuse. The first controlled thermal equilibrium was reached in a stellarator, a torus filled with deuterium, at a temperature of $800 \mathrm{eV}$ (10 million degrees) where fusion reactions were measured. ${ }^{4}$ This was made possible by the invention of the zeta (later theta) pinch, a plasma confinement system that uses an electric current in the plasma to generate a magnetic field that compresses the plasma. However, the diffusion of the plasma against the confining magnetic field was 20 times faster than expected by classical physics, which could be explained by a linear quantum correction factor of multiples of $40 \mathrm{eV}$ [see equation (2.46) of Ref. 5]. The measured thermal conduction parallel to the magnetic field ${ }^{6}$ exhibited exactly the same linear factor for the quantum correction of the collisions.

The temperature was later increased by a factor of almost 4 in the Wendelstein experiment that cost about one billion US\$. ${ }^{7}$ Similar temperatures of about 50 million degrees were reached in tokamak experiments such as ITER, but sufficiently long operation times that would be required for a commercial reactor are not expected to be reached for another 20 years. ${ }^{8}$ The NASEM report ${ }^{1}$ suggests that this process could be gradually accelerated by using better superconducting magnetic fields and by enforcing a coordinated collaboration of research between academia and industry. Still, the main challenge remains: the operation temperature of the plasmas must still be extreme, in the order of many tens of million Kelvin, similar to the sun or as in the Wendelstein experiment. ${ }^{4}$ As mentioned earlier, this is expected because the energy gains are not the result of chemical reactions but are coming from nuclear reactions.

The key role of nuclear fusion as a future basic energy source as recommended by the NASEM initiative includes the encouragement to engage in a wider range of alternative controlled nuclear fusion energy research to complement advances in magnetic confinement. One particularly promising approach is the application of a particular type of laser that can produce electric fields with peak power levels in the Petawatt (PW) range, so-called chirped-pulse amplifier systems (CPAs). This enables the generation of extreme radiation pressures that are fundamentally different from those generated by heating, as was demonstrated by a team that included the inventor of the CPA technique, Gerard Mourou. ${ }^{9}$ An advanced and innovative scheme that utilizes this approach for direct electricity generation was very recently proposed by one of the authors and was subsequently granted a US patent. ${ }^{10}$

\section{Nonthermal Pressures Generated by Laser Pulses}

The fact that the collective scientific national academy of the United States has come to the clear conclusion that nuclear fusion energy research must play a key role in the search for a solution 


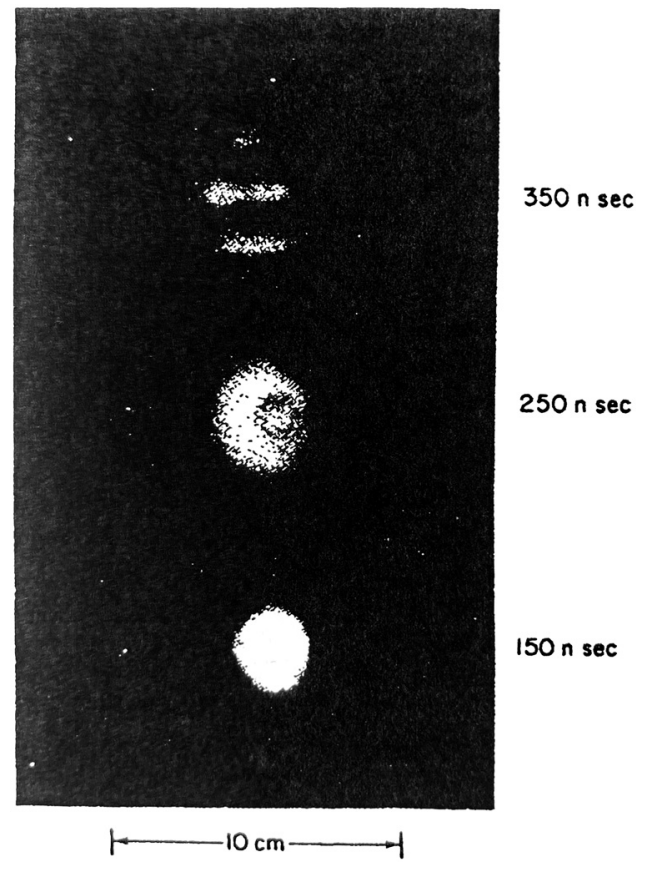

Fig. 1 Side-on framing camera picture of the plasma produced from an aluminum sphere of $80 \mu \mathrm{m}$ radius at discrete times after irradiation from the left by a 30 -ns long laser pulse from a ruby laser that was focused to a diameter of $0.4 \mathrm{~mm}$. The inner spherical part shows a plasma of 10 to $50 \mathrm{eV}$ temperature with the expected thermal expansion according to classical hydrodynamics. The second frame shows the outer part of a rapidly expanding plasma with ion energies of up to $5 \mathrm{keV} .^{12,13}$

for the world's energy problems in the future is of fundamentally crucial importance. The next question is whether this can be achieved without the need to heat the fuel to extreme temperatures in the order of hundreds of millions of degrees and to maintain it at these levels for a long duration. While thermal energy is one form of excitation energy, there are other nonthermal energy sources available that can provide the required forces or pressures. The extreme electric field strengths that can be produced with modern large-scale femtosecond or picosecond CPA laser systems have potential to provide a solution to the "hundred-million-degree temperature problem."11

Figure 1 shows a side-on view of a laser-irradiated free falling aluminum target in the focus of a laser beam at different times after excitation. ${ }^{12}$ One can see-and this was confirmed in hundreds of similar measurements as part of the same experiment-that the spherical central core behaves as classically expected. However, on top a half-moon-like plasma can clearly be observed with much higher ion energies that originate from a nonthermal acceleration. The generally accepted plasma equations of motion therefore had to be augmented by nonlinear terms $^{14,15}$ to result in a general equation of motion (summarized in Ref. 16) for the force density that can be written as

$$
f=-\nabla p+f_{N L}
$$

This equation contains the plasma-dynamic pressure $p$ with density $\rho$ and temperature $T$, whereas the nonlinear term-apart from a minor temperature dependence of the optical constant $n$-is determined by the electric and magnetic fields $E$ and $H$ that are contained in Maxwell's stress tensor $M$ via $f_{N L}=\nabla \bullet M .^{11}$ The specific contributions of each of those effects to the nonthermal acceleration can be seen from the full tensorial formulation of the complete transient nonlinear force (equation 8.88 in Ref. 5): 


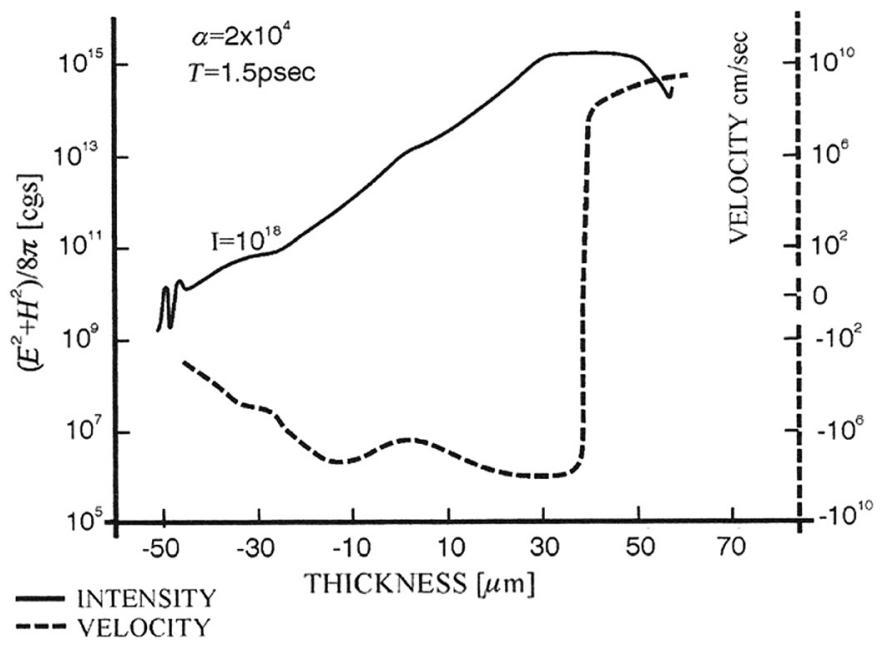

Fig. 2 Full hydrodynamically correct simulation for a neodymium glass laser with an intensity of $10^{18} \mathrm{~W} / \mathrm{cm}^{2}$ incident from the right-hand side onto an initially $100 \mathrm{eV}$ hot and very low reflecting biRayleigh deuterium plasma profile, shown at a time $t=1.5 \mathrm{ps}$. The figure contains the velocity distribution as a function of depth $(\boldsymbol{v}(\boldsymbol{x})$, dashed line) and the energy density of the laser field (solid line $(E 2+H 2) / 8 \pi)$. The laser-plasma interplay led to an acceleration of the deuterium plasma block of about 20 vacuum wavelength thickness of the dielectric enlarged skin layer moving against the laser and another block into the plasma showing ultrahigh $>10^{20} \mathrm{~cm} / \mathrm{s}^{2}$ acceleration, see Fig. 8.6. ${ }^{18}$

$$
f_{N L}=\nabla \cdot\left(E E+H H-\frac{1}{2}\left(E^{2}+H^{2}\right)\right)+\frac{\left(1+\frac{1}{\omega} \frac{\partial}{\partial t}\right)\left(n^{2}-1 E E\right)}{4 \pi}-\frac{1}{4 \pi c} \frac{\partial}{\partial t} E \times H
$$

First-principle hydrodynamic numerical evaluations ${ }^{17}$ (PhD thesis of V.F. Lawrence UNSW Sydney, 1978, see Fig. 2) revealed a nonthermal acceleration with picosecond (ps) laser pulses that were in full agreement with measurements with sub-ps laser pulses with PW peak powers ${ }^{19}$ that were subsequently enabled by the invention of the CPA technique decades later. ${ }^{20}$ This nonthermal acceleration was also measured with ps/PW laser pulses (see Fig. 4 of Refs. 11 and 21) where the nonthermally accelerated protons were of a low temperature plasma, but the gain was 10,000 times higher than at thermal equilibrium. This is another example of how a nonthermal acceleration of the plasma was happening without the need for thermal pressures equivalent to millions of degrees.

\section{Fresnel-Type Recoil and Plasma Acceleration}

When an optical wave enters from air into a dielectric, its phase speed decreases from $c$ to $c / n$, where $n$ is the dielectric refractive index and its momentum increases from $p$ to $n p$. The conservation of momentum then implies that reflection must occur in accordance with Fresnel equations. If the medium is not a dielectric but a layer of plasma with a depth-dependent refractive index, i.e., an inhomogeneous medium, this reflection can be suppressed. In that case, since conservation of momentum must be respected, this implies a recoil (acceleration against the optical pulse) of the plasma that can be described by a nonlinear force term ${ }^{22}$ derived from Maxwell's stress tensor in agreement with Fresnel's equations.

This can be seen in the computations shown in Fig. 2, which illustrates both the intensity of the laser pulse (solid line) and the plasma velocity (dash line) as a function of depth (thickness) below the air/plasma interface. With reference to the caption, Fig. 2 clearly shows the recoil of the plasma against the laser pulse followed by a region where the plasma velocity reaches up to $1000 \mathrm{~km} / \mathrm{s}$ against the direction of the laser pulse, which is in excellent agreement with measurements. ${ }^{19}$ 
Thus, the main challenge of controlled nuclear fusion, i.e., the need to heat the fuel to tens of millions of degrees Celsius to generate electrical energy, can be sidestepped as was demonstrated experimentally due to the nonlinearity of Maxwell's stress tensor and the subsequent use of the CPA laser pulses with high intensities. ${ }^{11,23}$ Several facilities around the world exist that are capable of generating ultrashort laser pulse with energies in the hundreds of joules to kilojoules level, one example being the L4 Aton laser system in the Czech Republic. ${ }^{24}$ These pulses can be focused down to a focal volume in the order of $10^{-9}$ to $10^{-10} \mathrm{~cm}^{3}$, corresponding to energy densities in excess of $10^{12} \mathrm{~J} / \mathrm{cm}^{3}$ that have been achieved in the focus of extreme CPA laser pulses.

\section{Fusion of Hydrogen $\mathrm{H}$ with the 11-Isotope of Boron}

The reaction ${ }^{25}$

$$
\mathrm{H}+{ }^{11} \mathrm{~B}=3{ }^{4} \mathrm{He}+8.7 \mathrm{MeV}
$$

is an aneutronic nuclear (HB11) fusion process where-fortunately only stable nuclei are involved. Secondary reactions can produce neutrons of very low density and undesired radioactive radiation, but in this case the number is comparably very small, and the use of tin for neutron capture and thermalizing can be used ${ }^{26}$ to realize electricity generators whose radioactivity can be kept well below the tolerable limit. This is essentially different to the fusion of heavy (deuterium) and super-heavy (tritium) hydrogen, on which the DT-fusion at low plasma density and magnetic field confinement is based as described in the first section of the NASEM report. ${ }^{1}$ Breeding, storing, and handling of tritium is a problem and during each fusion reaction, one neutron is generated. Still, the DT-fusion is the most straightforward fusion process, and the NASEM report recommends pursuing this option if no other viable solution emerges.

The difficulty of the hydrogen-boron (HB11) fusion can be seen if it is applied to the case of high-density fuel spheres that are compressed and heated prior to fusion as it is aimed for DT fusion (see Chapter 9 of Ref. 18). The highest fusion gains ever reported in scientific literature for DT with a breakeven of power gained per invested power is about $0.01,{ }^{27}$ although media reports have indicated that 0.7 has recently been achieved at the National Ignition Facility. ${ }^{28}$ Computations for HB11 (Ref. 18, Section 9.6) would require a compression of the fuel by more than five orders of magnitude and laser pulses of more than $100 \mathrm{MJ}$ of energy to achieve breakeven.

A radical change of this situation emerges with the use of laser pulses with a duration in the subpicosecond range as can be generated from CPA laser systems with peak powers in the order of several PW. As shown in Fig. 3 (taken from Ref. 29), the number of alpha particles generated per steradian has been continuously increasing and is further climbing (number of alpha particles

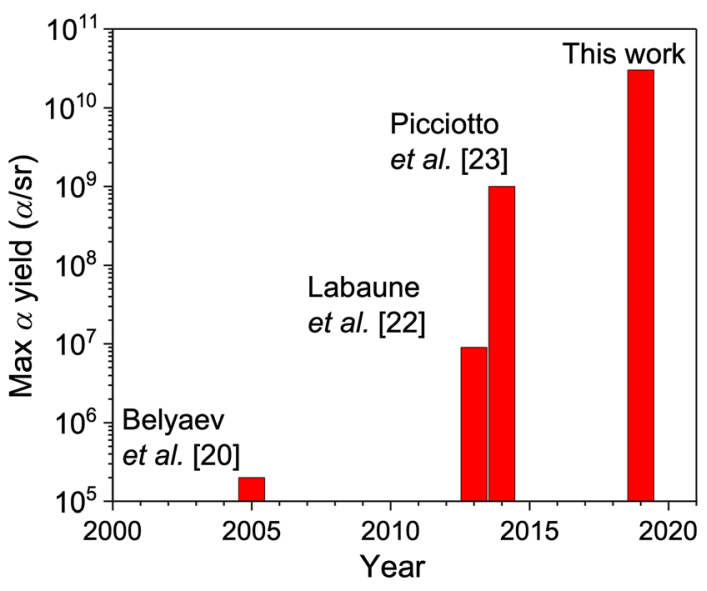

Fig. 3 Progression of the most important experimental achievements reported in the literature in terms of maximum $\alpha$ particles/sr (taken from Ref. 29). 
in brackets): $2005\left(10^{5}\right), 2013\left(10^{7}\right), 2014\left(10^{9}\right),{ }^{30}$ and $2019\left(4 \times 10^{10}\right)$. The result in 2014 was then used to trigger an avalanche of the generated $2.9 \mathrm{MeV}$ energetic alpha particles. The avalanche process was very precisely explained by elastic and nuclear collisions. ${ }^{31}$ However, Belloni et al. later demonstrated that the occurrence of avalanche reactions is not required to explain the experimental results obtained in $2014 .^{32} \mathrm{~A}$ concept reactor was developed including a spherical container where the energetic particles fly toward a positively charged electrode that is kept at a voltage of $1.4 \mathrm{MV}$, directly converting their nuclear energy into an electric current. ${ }^{33}$ The reactor design for the picosecond excitation pulses needed a cylindrical fuel container with magnetic trapping in a field of a few kiloTesla (kT). Such extreme magnetic fields were demonstrated in laser driven capacitor-coil geometries by Fujioka. ${ }^{34}$

\section{Conclusions}

These latest developments in laser-driven hydrogen-boron fusion are exciting and give reason for optimism. According to the NASEM report, ${ }^{1}$ nuclear fusion is one of the most promising future technologies to solve our planet's energy-related climate problem. Nuclear fusion can deliver 10 million times higher energies per reaction compared with chemical energy from burning coal. However, the greatest challenge is - and always has been-how to sustain a nuclear reaction under thermal equilibrium conditions at temperatures of tens of hundred million degrees, using purely thermal processes.

Our results show that nuclear fusion is also possible with a nonthermal ignition process, utilizing pressures produced by extreme CPA laser pulses. ${ }^{11}$ This is fundamentally different from fusion of low-density plasma in thermal equilibrium and thus opens up another avenue for a shift away from carbon energy toward nuclear energy via hydrogen-boron fusion.

The next steps will concentrate on the use of established PW CPA laser pulses to study the interaction and target properties ${ }^{35}$ with HB11 fuel in great detail. The development of a commercially viable HB11 reactor would offer a clean solution for energy generation, also in view of the radioactivity problem. ${ }^{26}$

\section{Acknowledgments}

The authors thank support of HB11 Energy Holdings Pty Ltd., especially with Lukasz Gadowski and Jan Kirchhoff, and to the support by Professor Claude Phipps, Santa Fe, NM, for supporting an electronic presentation of the summarized results at this HPLA (High Power Laser Ablation) virtual workshop that had to be managed on April 13, 2021, because of the present virus restrictions. The authors also thank Professor Vitaly Gruzdev for his helpful suggestions.

\section{References}

1. National Academies of Sciences, Engineering, and Medicine (NASEM), Bringing Fusion to the U.S. Grid, The National Academies Press, Washington, DC (2021).

2. World Energy Model Documentation, 2020 Version, Last Updated 7 May 2021, International Energy Agency (2021).

3. International Energy Outlook, U.S. Energy Information Administration Office of Integrated Analysis and Forecasting and U.S. Department of Energy, Washington, DC, 20585 (2010).

4. W VII-A TEAM and NI TEAM, "Plasma physics and controlled nuclear fusion research," in Proc. 8th Int. Conf. Brussels, Vol. 1, IAEA, Vienna, p. 185 (1981).

5. H. Hora, Plasmas at High Temperature and Density, Springer, Heidelberg (1991).

6. K. A. Razumova, "Results from T-7, T-10, T-11 and TM-4 Tokamaks," Plasma Phys. Control. Fusion 26, 37 (1984).

7. I. Milch, Tokamak und Stellarator - zwei Wege zur Fusionsenergie: Fusionsforschung," Phys. Unserer Zeit 37, 170 (2006).

8. B. J. Green, "The status of ITER - the fusion reactor development project," Aust. Phys. 55(4), 131 (2018). 
9. H. Hora et al., "Fusion energy using avalanche increased boron reactions for block-ignition by ultrahigh power picosecond laser pulses," Laser Part. Beams 33, 607 (2015).

10. H. Hora, "Method for generating electric energy by laser-based nuclear fusion and laser reactor," US Patent 10,410,752 (2019).

11. H. Hora et al., "Pressure of picosecond CPA laser pulses substitute ultrahigh thermal pressures to ignite fusion," High Energy Density Phys. 35, 100739 (2020).

12. E. W. Sucov et al., "Plasma production by a high-power Q-switched laser," Phys. Fluids 10(9), 2035 (1967).

13. H. Hora, Physics of Laser Driven Plasmas, Wiley, New York (1981).

14. H. Hora, "Nonlinear confining and deconfining forces associated with the interaction of laser radiation with plasma," Phys. Fluids 12, 182 (1969).

15. H. Hora, "The transient electrodynamic forces at laser-plasma interaction," Phys. Fluids 28, 3705 (1985).

16. T. P. Rowlands, "General foundation for the nonlinear ponderomotive four-force in laserplasma interactions," Laser Part. Beams 24, 475 (2006).

17. H. Hora, R. Castillo, and R. G. Clark, "Calculations of inertial confinement fusion gains using a collective model for reheat, Bremsstrahlung and fuel depletion for highly efficient electrodynamic laser compressions," Int. Atomic Energy Agency 3, 237 (1979).

18. H. Hora, Laser Plasma Physics, 2nd ed., SPIE Books, Bellingham, Washington (2016).

19. R. Sauerbrey, "Acceleration in femtosecond laser-produced plasmas," Phys. Plasmas 3, 4712 (1996).

20. D. Strickland and G. Mourou, "Compression of amplified chirped optical pulses," Opt. Commun. 56, 219 (1985).

21. P. A. Norreys et al., "Neutron production from picosecond laser irradiation of deuterated targets at intensities of $1019 \mathrm{~W} / \mathrm{cm}^{2}$," Plasma Phys. Control. Fusion 40(2), 175 (1998).

22. H. Hora, "Momentum of photons and the nonlinear force of laser-plasma interaction," Phys. Fluids 17, 1042 (1974).

23. S. Steinke et al., "Efficient ion acceleration by collective laser-driven electron dynamics with ultra-thin foil targets," Laser Part. Beams 28, 215 (2010).

24. https://www.eli-beams.eu/facility/lasers/laser-4-aton-10-pw-2-kj/.

25. M. L. E. Oliphant and E. Rutherford, "Experiments on the transmutation of elements by protons," Proc. R. Soc. London Ser. A 141(843), 259 (1933).

26. H. Hora, S. Eliezer, and N. Nissim, "Elimination of secondary neutrons from laser protonboron fusion," Laser Part. Beams 2021, 9978899 (2021).

27. O. Hurricane and D. H. Callahan, "Fuel gain exceeding unity in an inertially confined fusion implosion," Nature 506, 343 (2014).

28. https://www.llnl.gov/news/national-ignition-facility-experiment-puts-researchersthreshold-fusion-ignition.

29. L. Giuffrida et al., "High-current stream of energetic $\alpha$ particles from laser-driven protonboron fusion," Phys. Rev. E 101, 013204 (2020).

30. A. Picciotto et al., "Boron-proton nuclear-fusion enhancement induced in boron-doped silicon targets by low-contrast pulsed laser," Phys. Rev. X 4, 031030 (2014).

31. S. Eliezer et al., "Avalanche proton-boron fusion based on elastic nuclear collisions," Phys. Plasmas 23, 050704 (2016).

32. F. Belloni et al., "On the enhancement of p-11B fusion reaction rate in laser-driven plasma by $\alpha \rightarrow$ p collisional energy transfer," Phys. Plasmas 25, 020701 (2018).

33. H. Hora et al., "Laser boron fusion reactor with picosecond petawatt block ignition," IEEE Trans. Plasma Sci. 46, 1191 (2018).

34. S. Fujioka et al., "Kilotesla magnetic field due to a capacitor-coil target driven by high power laser," Sci. Rep. 3, 1170 (2013).

35. H. Hora and W. McKenzie, "Inhomogeneous dielectric target properties for increased nonthermal pressure in laser boron fusion by CPA-pulses," J. Energy Power Eng. 14, 273-278 (2020).

Heinrich Hora received his $\mathrm{PhD}$ from the University of Jena and his higher doctorate degree from the University of New South Wales (UNSW). He has been professor in theoretical physics 
with UNSW and is an Emeritus since 1992. He is an FInstP (London), fellow of the Australian Institute of Physics, and FRSN. He received the Ritter von Gerstner Medal, the Edward Teller Medal, the Dirac Medal, and the Ernst Mach Medal.

Alex Fuerbach received his MEng and PhD degrees from Vienna University of Technology. He worked for the company Femtolasers in Austria before moving to Australia in 2004. In late 2005, he was awarded an Australian Postdoctoral Fellowship which allowed him to establish his own research group at Macquarie University in Sydney, where he has been ever since. He is currently an associate professor and higher degree research director within the Department of Physics and Astronomy.

Francois Ladouceur is an internationally recognized researcher in the area of photonics, more specifically in integrated optics and optical sensing networks. His career straddled both academia and the private sector and focuses on applied research complemented with important commercialisation activities. He received his BEng and a MEng degrees both from École Polytechnique de Montréal and graduated with a $\mathrm{PhD}$ in optical telecommunication from the Australian National University (ANU).

Warren McKenzie is a science entrepreneur, materials scientist, endeavour fellow, and fellow of the Royal Society of New South Wales. He has held research positions at Trinity College in Dublin and at UNSW. He has considerable experience establishing large scientific collaborations and transforming them into companies. He has been a finalist for the prestigious 2020 Young Entrepreneur of the Year Award conducted by Business News Australia, and he is currently managing director of HB11 Energy Holdings. 